\title{
Internal magnetic field effect on magnetoelectricity in orthorhombic $R \mathrm{MnO}_{3}$ crystals
}

\author{
K. Noda*, M. Akaki, F. Nakamura, D. Akahoshi, H. Kuwahara \\ Department of Physics, Sophia University, 7-1 Kioi-cho, Chiyoda-ku, Tokyo 102-8554, Japan.
}

\begin{abstract}
We have investigated the role of the $4 f$ moment on the magnetoelectric (ME) effect of orthorhombic $R$ MnO ${ }_{3}(R=$ rare earth ions). In order to clarify the role of the $4 f$ moment, we prepared three samples: $(\mathrm{Eu}, \mathrm{Y}) \mathrm{MnO}_{3}$ without the $4 f$ moment, $\mathrm{TbMnO} \mathrm{O}_{3}$ with the anisotropic $4 f$ moment, and $(\mathrm{Gd}, \mathrm{Y}) \mathrm{MnO}_{3}$ with the isotropic $4 f$ moment. The ferroelectric behaviors of these samples are different from each other in a zero magnetic field. $(\mathrm{Eu}, \mathrm{Y}) \mathrm{MnO}_{3}$ and $(\mathrm{Gd}, \mathrm{Y}) \mathrm{MnO}_{3}$ show the ferroelectric polarization along the $a$ axis in the ground state, while $\mathrm{TbMnO}_{3}$ shows it along the $c$ axis. Such difference may arise from the influence of the anisotropic $\mathrm{Tb}^{3+}$ $4 f$ moment. The direction of the ferroelectric polarization of $R \mathrm{MnO}_{3}$ is determined by the internal magnetic field arising from the if moment.
\end{abstract}

(C) 2018 Elsevier B.V. All rights reserved.

PACS: 75.80.+q; 71.45.Gm; 77.84.Bw; 77.80.Fm; 75.30.-m; 75.50.Ee;

Keywords: Multiferroics; Magnetoelectric effect; Ferroelectrics; Antiferromagnetism

A strong correlation between dielectric and magnetic properties, so called magnetoelectric effect (ME), has attracted a revived interest. The series of orthorhombic $R \mathrm{MnO}_{3} \quad\left(R=\right.$ rare earth ions) containing $\mathrm{TbMnO}_{3}$ famous as the "magnetic-field-induced electric polarization flop" [1] is a parent material of the colossal magnetoresistance (CMR) manganite. Recently, the noncollinear transverse spiral antiferromagnetic (AF) order of Mn $3 d$ spins is observed between the $A$-type $\left(\mathrm{EuMnO}_{3}\right)$ and the E-type $\left(\mathrm{HoMnO}_{3}\right)$ AF order.[2,3,4] Such unconventional magnetic order is caused by the competition between the ferromagnetic nearest neighbor $(\mathrm{NN})$ interaction and the AF next NN interaction due to the relatively large orthorhombic distortion. Such noncollinear transverse spiral magnetic structure causes the ferroelectric polarization of $R \mathrm{MnO}_{3} \cdot[3,4,5,6]$

In previous work, we have investigated the ME effect in $\left(\mathrm{Eu}_{0.595} \mathrm{Y}_{0.405}\right) \mathrm{MnO}_{3}\left((\mathrm{Eu}, \mathrm{Y}) \mathrm{MnO}_{3}\right)$ without $4 f$ moment.[7] Even if the average ionic radius of $R$ site in $(\mathrm{Eu}, \mathrm{Y}) \mathrm{MnO}_{3}$ is the same as that of $\mathrm{TbMnO}_{3}$, the direction of the ferroelectric polarization of the ground state of $(\mathrm{Eu}, \mathrm{Y}) \mathrm{MnO}_{3}$ is different from that of $\mathrm{TbMnO}_{3}$ in a zero magnetic field: $(\mathrm{Eu}, \mathrm{Y}) \mathrm{MnO}_{3}$ shows the ferroelectric polarization along the $a$ axis $\left(P_{a}\right)$, while $\mathrm{TbMnO}_{3}$ shows

\footnotetext{
* Corresponding author. Tel.:+81-3-3238-3399;Fax:+81-3-3238-3430 Email address: n-kohei@sophia.ac.jp (K. Noda).
}

$P_{c}$. It is considered that this difference is attributed to the magnetic property of each $R$ ion.

In this paper, we have revealed the role of the rare-earth $4 f$ moment on the ME effect of orthorhombic $R \mathrm{MnO}_{3}$. In order to investigate the role of the $4 f$ moment, we prepared three samples: $(\mathrm{Eu}, \mathrm{Y}) \mathrm{MnO}_{3}$ without the $4 f$ moment, $\mathrm{TbMnO}_{3}$ with the anisotropic $4 f$ moment, and $\left(\mathrm{Gd}_{0.69} \mathrm{Y}_{0.31}\right) \mathrm{MnO}_{3}\left((\mathrm{Gd}, \mathrm{Y}) \mathrm{MnO}_{3}\right)$ with the isotropic $4 f$ moment. The average ionic radius of $R$ site in these samples is fixed to be same as that of $\mathrm{TbMnO}_{3}$.

The compositional ratio of the $\mathrm{Eu}^{3+}$ to $\mathrm{Y}^{3+}$ ions and that of $\mathrm{Gd}^{3+}$ to $\mathrm{Y}^{3+}$ were obtained by a calculation based on the Shannon's ionic radius table. [8] The single crystal sample was grown by the floating zone method. We performed $\mathrm{x}$-ray-diffraction and rocking curve measurements on the resulting crystal at room temperature, and confirmed that the samples have the orthorhombic Pbnm structure without any impurity phases or any phase segregation. All specimens used in this study were cut along the crystallographic principal axes into a rectangular shape by means of x-ray back-reflection Laue technique. The measurement of the temperature dependence of the spontaneous ferroelectric polarization was carried out in a temperature-controllable cryostat. The spontaneous electric polarization was obtained by the accumulation of a pyroelectric current. The magnetization was measured using a commercial apparatus (Quantum Design, PPMS). 

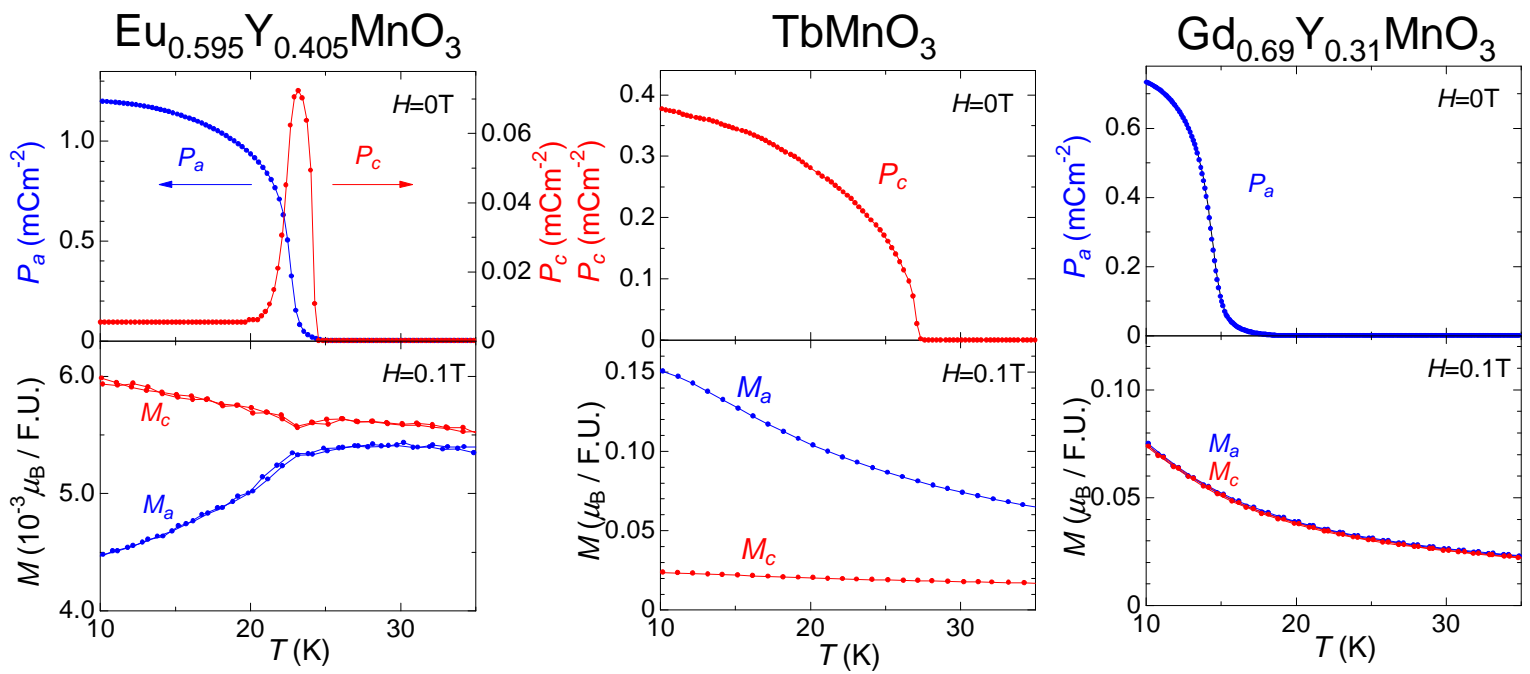

Fig. 1. Temperature dependence of the ferroelectric polarization (upper) and magnetization (lower) of the $(\mathrm{Eu}, \mathrm{Y}) \mathrm{MnO}_{3}\left(\mathrm{left}\right.$ panel), $\mathrm{TbMnO}_{3}$ (center panel), and (Gd,Y) $\mathrm{MnO}_{3}$ (right panel).

At first, we focus on $(\mathrm{Eu}, \mathrm{Y}) \mathrm{MnO}_{3}$ without $4 f$ moment. In a zero magnetic field, this compound shows two distinct ferroelectric phases with $P_{a}(T \leq 23 \mathrm{~K})$ and $P_{c}(23 \mathrm{~K} \leq T \leq 25 \mathrm{~K})$ (See Fig.1 left panel). The magnitude of the magnetization is about $10^{-3} \sim 10^{-4} \mu_{\mathrm{B}} /$ F.U. in $H=0.5 \mathrm{~T}$. The behavior of magnetization is directly reflecting the behavior of the $\mathrm{Mn}$ $3 d$ spins, because this compound is free from $4 f$ moment. In previous work, we have demonstrated the ferroelectric polarization switching between $P_{a}$ and $P_{c}$ by application of the magnetic field: For example, the $P_{a}$ changes to the $P_{c}$ by the application of the magnetic field parallel to the $a$ axis $\left(H_{a}^{\mathrm{ext}}\right)$. [7] This switching behavior is caused by the flop of the transverse spiral AF plane of Mn $3 d$ spins, which can be explained in terms of the inverse Dzyaloshinskii-Moriya interaction. [6]

In the case of $\mathrm{TbMnO}_{3}$, the dielectric and magnetic properties are different from those of $(\mathrm{Eu}, \mathrm{Y}) \mathrm{MnO}_{3}$, although the average ionic radius of $R$ site is the same (See Fig.1 center panel). $\mathrm{TbMnO}_{3}$ shows only $P_{c}$ in a zero magnetic field. The magnitude of the magnetization is about $10^{1} \sim 10^{2}$ times as large as that of $4 f$-moment free $(\mathrm{Eu}, \mathrm{Y}) \mathrm{MnO}_{3}$, because of the influence of the $\mathrm{Tb}^{3+} 4 f$ moment. Introducing the $4 f$ moment enhances the magnitude of the magnetization along the $a$ axis $\left(M_{a}\right)$ compared with the other axes. This result suggests that the $4 f$ moment of $\mathrm{TbMnO}_{3}$ is anisotropic, and the internal magnetic field due to the $4 f$ moment seems to be parallel to the $a$ axis $\left(H_{a}^{\text {int }}\right)$. In the case of $4 f$-moment free $(\mathrm{Eu}, \mathrm{Y}) \mathrm{MnO}_{3}$, the ground state has $P_{a}$, and the direction of the ferroelectric polarization is changed from the $a$ axis to the $c$ axis by the application of $H_{a}^{\text {ext }}$, as mentioned above. Therefore, in $\mathrm{TbMnO}_{3}, P_{c}$ is stabilized by the application of $H_{a}^{\text {int }}$, like the case of $4 f$-moment free $(\mathrm{Eu}, \mathrm{Y}) \mathrm{MnO}_{3}$ in $H_{a}^{\mathrm{ext}}$. Hence, the difference of the direction of the ferroelectric polarization between $4 f$-moment free $(\mathrm{Eu}, \mathrm{Y}) \mathrm{MnO}_{3}$ and anisotropic $4 f$-moment $\mathrm{TbMnO}_{3}$ can be understood from the scenario that $H_{a}^{\text {int }}$ acts as $H_{a}^{\text {ext }}$.
In the case of $(\mathrm{Gd}, \mathrm{Y}) \mathrm{MnO}_{3}$, the $P_{a}$ only appears in a zero magnetic field (See Fig.1 right panel). The magnitude of the magnetization is nearly the same as that of $\mathrm{TbMnO}_{3}$. However, in this compound, no significant difference is observed between the temperature dependence of $M_{a}$ and $M_{c}$, in contrast to the case of $\mathrm{TbMnO}_{3}$. Therefore, the $4 f$ moment of this compound is isotropic. As a result, the effective internal magnetic field does not exist. Consequently, the direction of the ferroelectric polarization of this compound is the same as that of $4 f$-moment free $(\mathrm{Eu}, \mathrm{Y}) \mathrm{MnO}_{3}$.

From these results, we conclude that, in $R \mathrm{MnO}_{3}$ crystals, the Mn $3 d$ spins are indispensable for realizing the ferroelectric polarization, while the $4 f$ moment is not. The magnetic easy axis of the $4 f$ moment determines the direction of the ferroelectric polarization through the channel of the internal magnetic field, even in a zero external magnetic field. The results obtained in this experiment should provide an improved understanding of the mechanism of the magnetoelectric effect in $R \mathrm{MnO}_{3}$ crystals.@

\section{References}

[1] T. Kimura, T. Goto, H. Shintani, K. Ishizaka, T. Arima, and Y. Tokura, Nature 426 (2003), p. 55.

[2] T. Kimura, S. Ishihara, H. Shintani, T. Arima, K. T. Takahashi, K. Ishizaka, and Y. Tokura, Phys. Rev. B 68 (2003), p. 060403(R).

[3] M. Kenzelmann, A. B. Harris, S. Jonas, C. Broholm, J. Schefer, S. B. Kim, C. L. Zhang, S.-W. Cheong, O. P. Vajk, and J. W. Lynn, Phys. Rev. Lett. 95 (2005), p. 087206.

[4] T, Arima, A. Tokunaga, T. Goto, H. Kimura, Y. Noda, and Y. Tokura, Phys. Rev. Lett. 96 (2006), p. 097202.

[5] H. Katsura, N. Nagaosa, and A. V. Balatsky, Phys. Rev. Lett. 95 (2005), p. 057205.

[6] M. Mostovoy, Phys. Rev. Lett. 96 (2006), p. 067601.

[7] K. Noda, M. Akaki, and T. Kikuchi, D. Akahoshi, and H. Kuwahara, J. Appl. Phys. 99 (2006), p. 08S905.

[8] R. D. Shannon, Acta Crystallogr. Sect. A 32 (1976), p. 751. 